\title{
Progress on Pharmaceutical Sciences/Pharmacy Postgraduate Education: a Bibliometric Perspective
}

\author{
Zhengwei Huang ${ }^{1,2} \cdot$ Xuejuan $\mathrm{Zhang}^{2} \cdot$ Linjing $\mathrm{Wu}^{2} \cdot$ Ping $\mathrm{Hu}^{2} \cdot$ Ying Huang ${ }^{2} \cdot \mathrm{Xin} \mathrm{Pan}^{1} \cdot$ Chuanbin $\mathrm{Wu}^{2}$
}

Accepted: 23 November 2021 / Published online: 22 January 2022

(c) The Author(s), under exclusive licence to Springer Science+Business Media, LLC, part of Springer Nature 2021

\begin{abstract}
Objective The study quantitatively investigated the related research progress in pharmaceutical sciences/pharmacy education from a bibliometric angle and provided feasible suggestions to facilitate the development of pharmaceutical sciences/ pharmacy postgraduate education.

Methods Bibliometric analysis was conducted using the database of Web of Science Core Collection. The literature published in 1985-2021 was screened and selected. The overall profile description, citation analysis, and research hotspot mining were performed using the citation report of Clarivate Analytics, bibliometrics online platform, and VOSviewer software. The bibliometric results and profiles were plotted and illustrated.

Results The bibliometric analysis of 485 papers of interest showed that the research frontier was continuously expanding; especially the institutions from the USA were the main contributors. The numbers of citing papers have been ascending, and a considerable part of citations were from the areas other than the education research. Mining results showed that the in-school and residency education of pharmacy postgraduates was a research hotspot, as well as interprofessional training and new education styles for Coronavirus Disease 2019 (COVID-19) therapy were the emerging trends in the field.

Conclusion Through the analysis of the studies, it was found that encouraging relevant research programs, establishing financial supports, and launching specified publication sources could be helpful to boost the development of pharmaceutical sciences/pharmacy postgraduate education. Besides, the results suggested that this was a less discussed topic and was worthy for the investigators to pay more attention to such an issue.
\end{abstract}

Keywords Bibliometric $\cdot$ Pharmaceutical sciences $\cdot$ Pharmacy $\cdot$ Postgraduate education $\cdot$ Web of science

\section{Introduction}

Nowadays, a high level of healthcare is one of the vital standards for the citizens in a modern society [1]. Ample supply and rational administration of medical products, especially

Zhengwei Huang, Xuejuan Zhang, Linjing Wu are contributed equally to this work.

Xin Pan

panxin2@mail.sysu.edu.cn

Zhengwei Huang

huangzhengw@jnu.edu.cn

Xuejuan Zhang

zhanghongdou0223@126.com

Linjing $\mathrm{Wu}$

wlinjing777@163.com

Ping $\mathrm{Hu}$

inzahu@ @otmail.com the drugs, are the prerequisites for such a standard [2]. In most countries, drug production/supply and administration are associated with pharmaceutical industry and pharmacist systems, respectively, which demand the teamwork of experts in both fields. Recently, in order to treat longterm diseases (e.g., hypertension, diabetes, etc.) and severe epidemics (e.g., Coronavirus Disease 2019, COVID-19),

Ying Huang

huangy2007@jnu.edu.cn

Chuanbin Wu

chuanbin_wu@126.com

1 School of Pharmaceutical Sciences, Sun Yat-Sen University, Guangzhou 510006, China

2 College of Pharmacy, Jinan University, Guangzhou 510006, China 
sustainable development of pharmaceutical industry and pharmacist system is of urgent need [3]. It is widely recognized that education is the primary approach to guarantee the sustainable development of a discipline. Research on education can favor the rational design of curricula, improve the evaluation systems and update the study zone for the students [4], and is therefore of great significance.

The research on postgraduate education is particularly important in the field of pharmaceutical sciences and pharmacy. Currently, emphases are laid on complex interactions in pharmaceutical industry and pharmacist system, which rely on, but not limited to, the abilities about scientific research, program management, self-education and disciplinary integration [5]. Undergraduate training is normally focused on the fundamental knowledge and skills in the field [6], while postgraduate education pays much attention to the medical or investigational cases, which played a critical part in the cultivation of more advanced skills and capabilities.

From this point, research on the progress and current status of pharmaceutical sciences/pharmacy postgraduate education is meaningful and necessary. In this paper, the relevant research progress was showcased in detail from a bibliometric perspective. The overall profile of the literature was outlined and the statistical analysis of citation was exhibited. Research hotspot mining was performed to analyze the evolution trend in this field. It was expected that this paper could provide valuable information and advices for the investigators interested in pharmaceutical sciences/ pharmacy education.

\section{Methods}

The scope of bibliometric analysis was in the Science Citation Index Expanded (SCI-EXPANDED) database of Web of Science Core Collection, so as to screen the relatively high-impacted papers in the field [7]. The search sets were (TOPIC $=$ pharmaceutical science postgraduate education) or $($ TOPIC $=$ pharmaceutical science graduate student education) or (TOPIC $=$ pharmaceutical science master student education) or (TOPIC $=$ pharmaceutical science doctoral student education) or $($ TOPIC $=$ pharmacy postgraduate education) or (TOPIC = pharmacy graduate student education) or (TOPIC $=$ pharmacy master student education) or (TOPIC $=$ pharmacy doctoral student education). The literature survey was completed at 17:00 (UST + 8), March $22^{\text {nd }}, 2021$, and the papers within the whole timespan (1985-2021) were included.

After removing the duplicated and the irrelevant hits, the residual papers were analyzed by a citation report of Clarivate Analytics [8], CC BY-NC-SA 4.0 bibliometrics online platform (https://bibliometric.com) [9] and VOSviewer software (version 1.6.16) [10].

\section{Results and Discussion}

\section{Overall Profile of the Literature}

The literature survey retrieved 485 papers (duplicated and irrelevant hits precluded) in total. Among them, 236 papers are from open access (OA) journals. The distributions of publication time, literature types, nationalities, institutions, journals, authors, grant sources, and languages were analyzed [11].

\section{Distribution of Publication Time}

The first 9 papers with the topic of pharmaceutical sciences/ pharmacy postgraduate education were published in 1999, indicating that the related research interest was formed around the millennium. From 2000 to 2004, the numbers of publications slightly dropped, and again raised after 2005 . In 2020, 50 papers were published. Generally, it was shown that the research interest was growing in the field. The tendency is demonstrated in Fig. 1.

The distribution profile suggested that the research of pharmaceutical sciences/pharmacy postgraduate education was an emerging area for exploration. Given the growing trend of the numbers of publications in the last decade, it was anticipated that more papers will be published in 2021 and the future.

\section{Distribution of Literature Type}

The literature was categorized into three types, viz. article, review, and others (Fig. 2A). 'Article' consisted of a proportion of $87.42 \%$, suggesting that most papers had provided new methodological, technological or analytical insights into the science community. The proportion of 'review' was quite low (6.80\%), even lower than 'others' (mainly refer to editorial materials which might not be fully regarded as scientific papers) [12]. From this perspective, summary and refinement of the previous studies lagged far behind the boosting tendency of the published articles in this field. It would be advisable for the researchers in this field to make more attempts to summarize and refine the published works. The institutions from the USA had published the largest numbers of literature and correspondingly, English is the dominant language for the authors to compose those papers, followed by Japanese and Spanish (Fig. 2B, C).

\section{Distribution of Countries/Regions}

Overall, researchers from 65 countries or regions had contributed their studies in this field. According to Fig. 3A, 


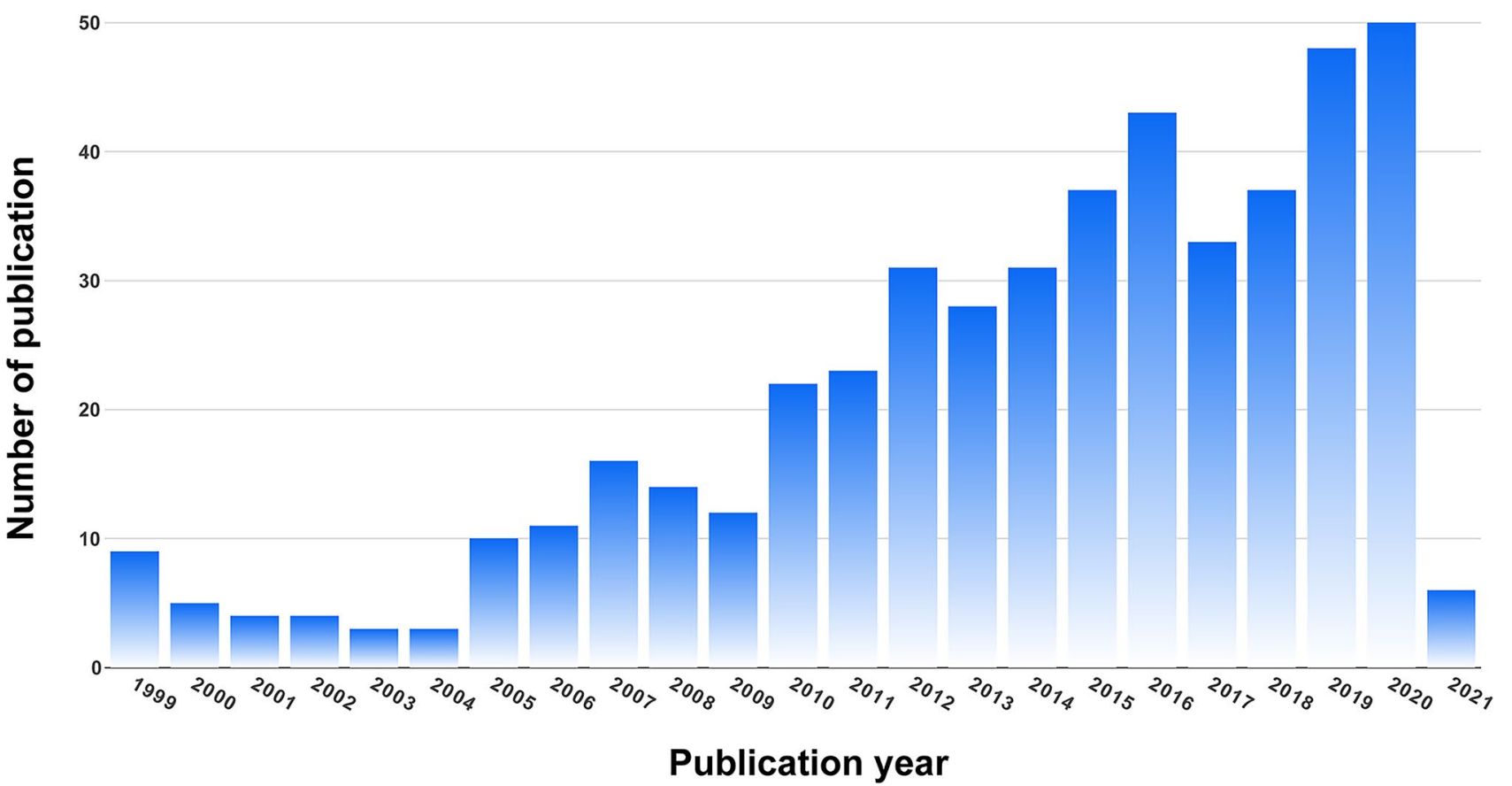

Fig. 1 Overall profile of the literature: distribution of time $(n=485)$

China, Australia and USA had participated in pharmaceutical sciences/pharmacy postgraduate education research in the earliest period, and the researchers in these countries continued to make their contributions. The researchers in Japan and UK started the related studies shortly after 1999, while the researchers in India, the Netherlands, Qatar, Saudi Arabia, and Canada joined the community from 2006.

The countries with higher numbers of publications $(\geq 10)$ are summarized in Fig. 3B. The USA published over 250 papers, while China, Saudi Arabia, Canada, Japan, the UK, Australia, and Qatar contributed 10-40 papers.

Evidently, the main countries contributing pharmaceutical sciences/pharmacy postgraduate education research papers were developed economics with substantial populations. In general, these countries had relatively higher budgets for national healthcare systems and would emphasize and realize the importance of the cultivation of pharmaceutical sciences/pharmacy graduates [13]. Moreover, good economic situations guaranteed the financial supports for such research [14], and sufficient amounts of postgraduates and related research could be expected from such countries with larger populations.

Furthermore, the cooperation relationship between countries/regions was visualized in Fig. 3C. The connecting curves represented the cooperation relationship, and the boldness of the curves indicated the numbers of the
A

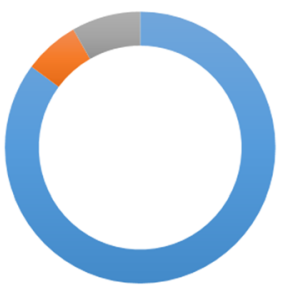

Article (424, 87.42\%)

Review $(33,6.80 \%)$

Others $(41,8.45 \%)$
B

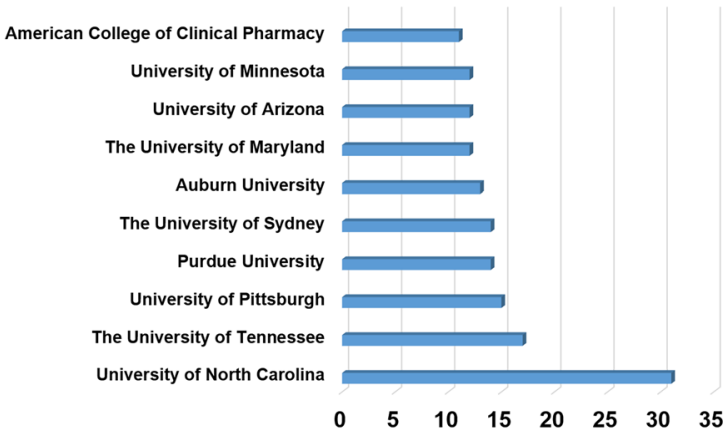

C

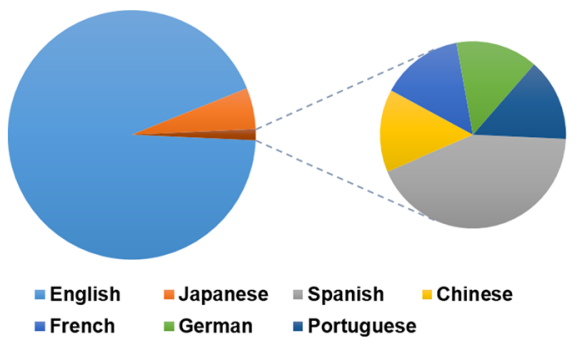

Fig. 2 Overall profile of the literature: distribution of literature types (A), institutions $(\mathbf{B})$, and languages $(\mathbf{C})(n=485)$ 
A

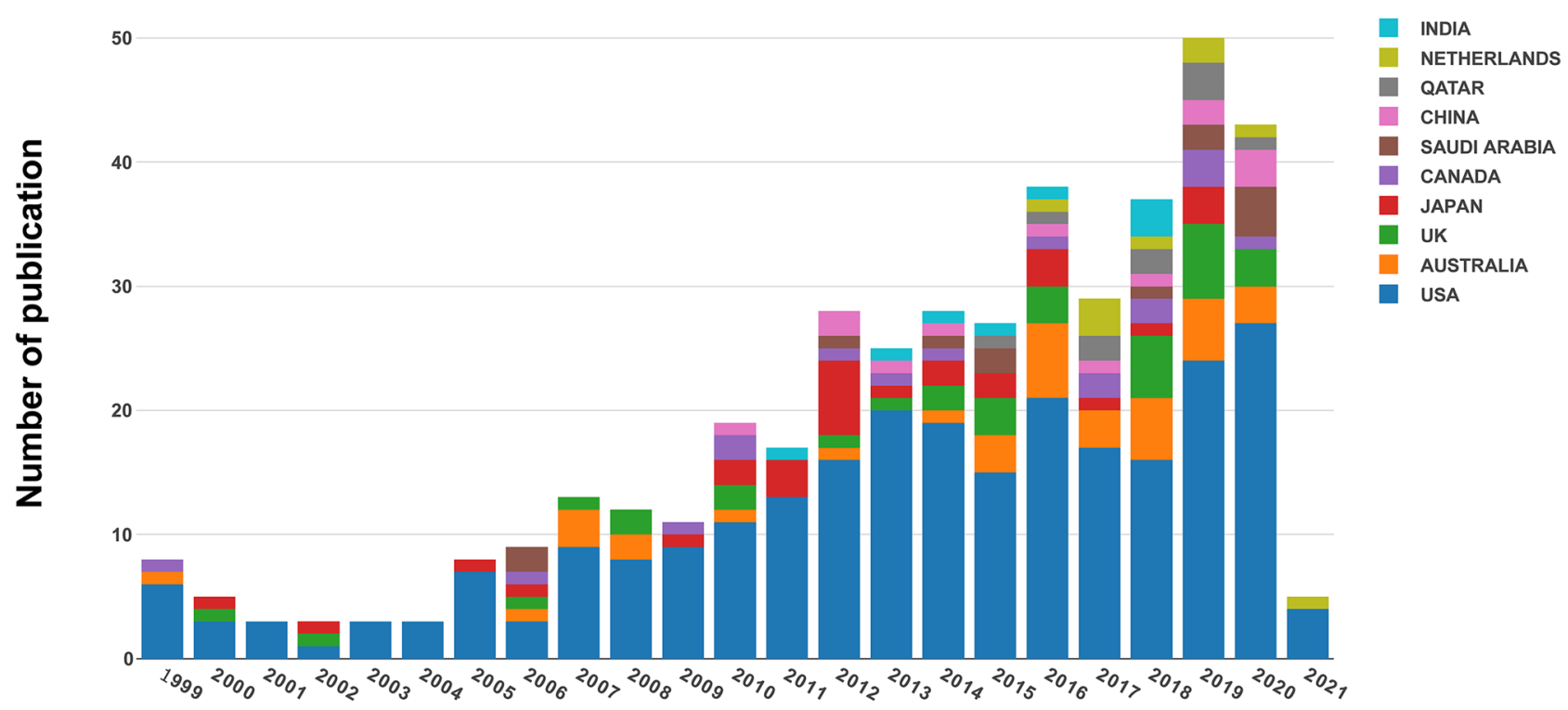

Publication year

B

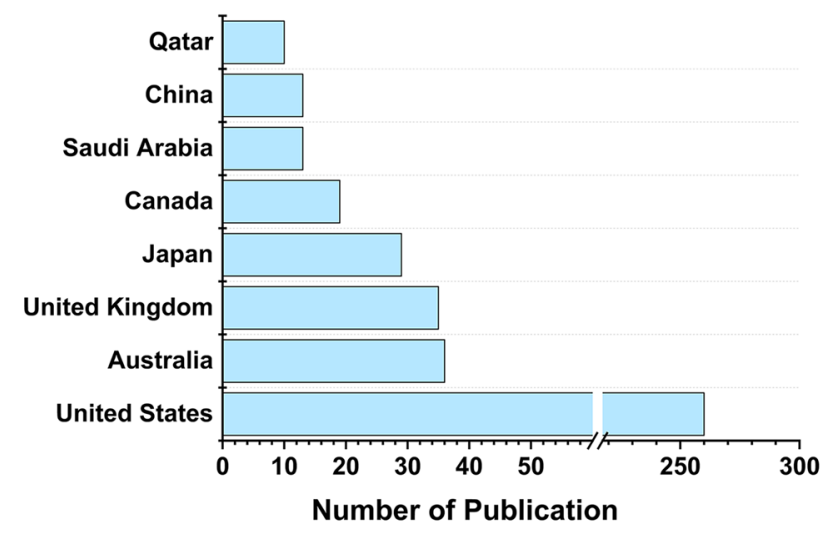

Fig. 3 Overall profiles of the literature: distributions of countries/ regions versus publication years (A), distributions of countries/ regions versus numbers of publications $(\mathbf{B})$, and cooperation relation-

cooperation activities [15]. For instance, there was a cooperation relationship between China and Pakistan; Thailand and UK formed a strong cooperative tile. Generally, the international cooperation was, to some extent, inactive. Many
C

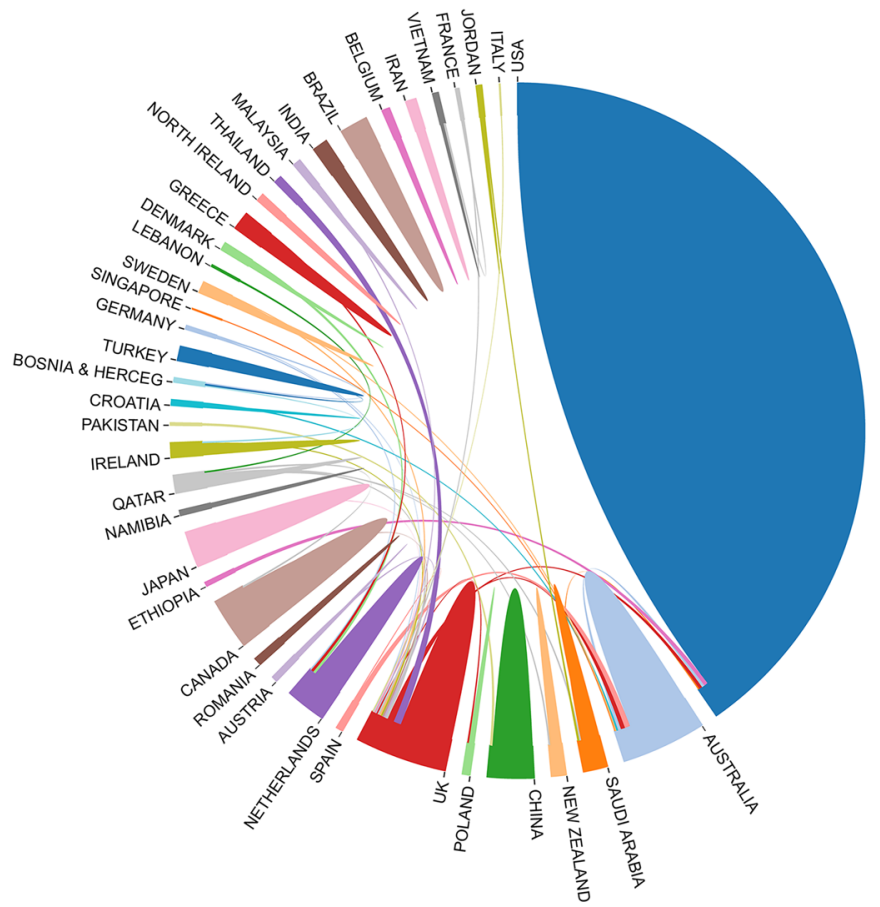

ship between the nations (C) $(n=485)$. Noticeably, only the main hits of countries/regions were listed herein

countries (like Brazil and India) completed the research individually, and the USA that credited the highest number of publications had only cooperated with four countries, i.e. Ethiopia, the UK, Saudi Arabia, and Australia. 
The reason for the relatively low cooperative activity might be explained as follows. The postgraduate education systems and the regulations thereof varied largely among countries [16], and thus there would be some technical barriers impeding the interactions. Consequently, for the countries that shared the same languages, similar cultures or neighboring geographical relations, the international cooperation would be easier and more feasible. This accounted for the cooperation between China and Pakistan, Qatar and Lebanon, Saudi Arabia and Jordan, USA and Australia, etc.

Albeit the technical barriers, it was suggested that more international cooperation could be generated, so as to boost the communication and the development of pharmaceutical sciences/pharmacy postgraduate education globally.

\section{Distribution of Institution}

The published papers were contributed by 693 institutions, and 671 of them $(96.83 \%)$ published less than 10 papers. The top-10 institutions with the largest numbers of contributions (11-31 papers) are displayed in Fig. 2B. Except for The University of Sydney (in Australia), the other 9 institutions were all affiliated to the USA. The University of North Carolina, The University of Tennessee, and the University of Pittsburgh were the top-3 contributing institutions, who credited 31,17 , and 15 papers, respectively.

Calculated by the numbers of institutions (693) and publications (485), there were 1.43 institutions averagely listed per paper. It suggested that a substantial part of papers were cooperated between two or more institutions. Compared with the international cooperation, the inter-institutional cooperation was rather active. It was further conjectured that a large proportion of the cooperating institutions were affiliated to the same countries. This was reasonable since the technical barriers for cooperation could be largely avoided in such a scenario.

\section{Distribution of Journals}

The papers were distributed in 133 journals. Considering the source of the analysis, those journals were all SCIEXPANDED indexed journals. The top-9 journals with more than 10 publications (295 papers in total, $60.82 \%$ of 485 papers) are showed in Fig. 4A. As a flagship journal in this field, the American Journal of Pharmaceutical Education included 136 papers, as $28.04 \%$ of all papers. The other professional journals about education, viz. Indian Journal of Pharmaceutical Education and Research, BMC Medical Education, and Journal of Chemical Education published 20, 14, and 13 papers, respectively. If adding those not shown in the chart, there were 202 papers published in educationspecified journals, accounting for a percentage of $41.65 \%$. In summary, a considerable part of pharmaceutical science/ pharmacy postgraduate education researches were published in education-specified journals.

The journals specified in healthcare systems, like the American Journal of Health System Pharmacy, Journal of Interprofessional Care, and Journal of the American Pharmacists Association, also held a certain proportion of the papers. A journal for fundamental research, Pharmacotherapy, included 16 papers. It was worth mentioning that Yakugaku Zasshi-Journal of the Pharmaceutical Association of Japan, a Japanese-language journal, included 26 papers.

It was found that education-specified, healthcare systemsrelated, and fundamental research-based journals were the major publishing platforms for relevant studies. Evidently, education-specified journals were the suitable media. As for healthcare system-related and fundamental research-based journals, they acted as the forums for the publications from the investigators in pharmacy postgraduate education and pharmaceutical sciences postgraduate education, respectively. Potentially, journals about psychology (particularly educational psychology) [17] would be an emerging publishing platform.
Fig. 4 Overall profile of the literature: distribution of periodicals (A) $(n=485)$; Citation analysis: distribution of periodicals of the cited papers (B) $(n=3264)$

\section{A}

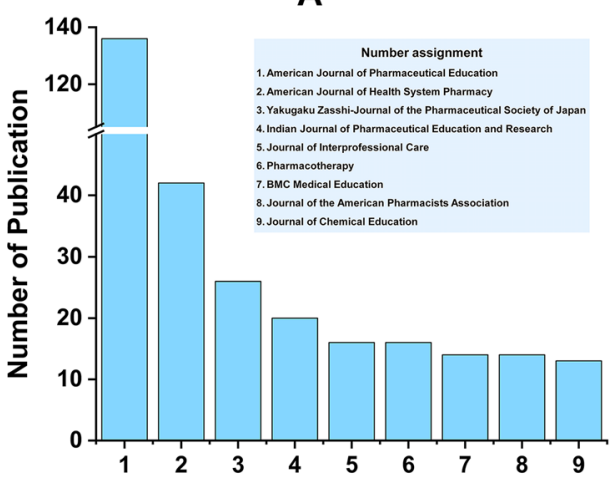

B

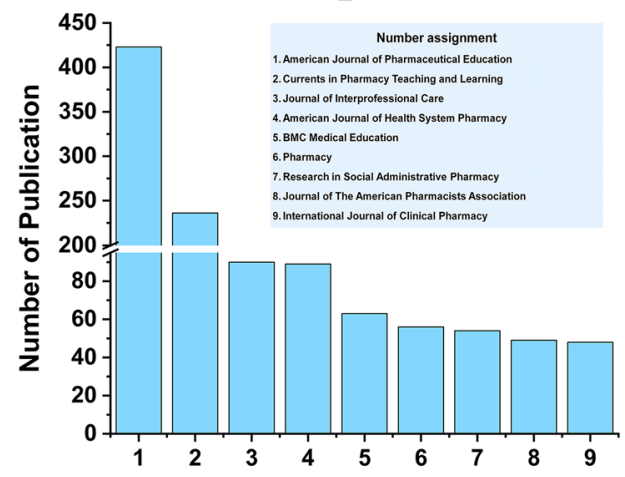




\section{Distribution of Author}

In the author-list of the papers, 1920 different names were recorded. Among them, Poloyac S.M. and Wilby K.J. (2 authors in total, $0.001 \%$ ) authored 5 papers, Anderson C., Awaisu A., etc. (13 authors in total, $0.007 \%$ ) authored 4 papers, Austin Z., Bush C.G., etc. (22 authors in total, $0.011 \%$ ) authored 3 papers, and Al-Arifi M.N., Alston G.L., etc. (119 authors in total, 6.198\%) authored 2 papers. Abadier M., Abanonu G.B., etc. (1764 authors in total, $91.875 \%$ ) authored 1 paper.

Calculating from the numbers of authors (1920) and publications (485), averagely 3.96 authors co-authored one paper, which confirmed co-authorization was a prevailing phenomenon in this field. The cooperation network of authors was analyzed. Poloyac S.M. and Wilby K.J., from the University of Pittsburgh (the USA) and University of Otago (New Zealand), respectively, both authored 5 papers, were taken as examples. Figure 5 showed that Poloyac S.M. and Wilby K.J. were the core of the cooperation network, and cooperated with 27 and 16 co-authors, respectively.

\section{Distribution of Grant Source}

The total number of grant sources reached 199, as 0.41 funding was received per paper in average. National
Institutes of Health, USA (NIH) and The United States Department of Health and Human Services (HHS) sponsored 23 and 21 papers, respectively. It could be inferred that NIH and HHS laid emphasis on this field. The other sources sponsored no more than 5 papers. The National Natural Science Foundation of China (NSFC), China Medical Board and Professional Committee of Medical Education, Chinese Academy of Higher Education sponsored 2, 2, and 1 paper (5 papers in total), respectively.

It was worth noticing that only 138 papers were sponsored by at least one source $(28.45 \%)$, and the funding information was not available for 347 papers $(71.55 \%)$. It suggested that major part of papers was without sponsorships and more financial supports should be provided to facilitate the investigations.

\section{Citation Situations}

The citation of a publication was a key indicator of the attractiveness and importance of the study [18]. For further bibliometric analysis, the citation situations of the 485 papers were analyzed.

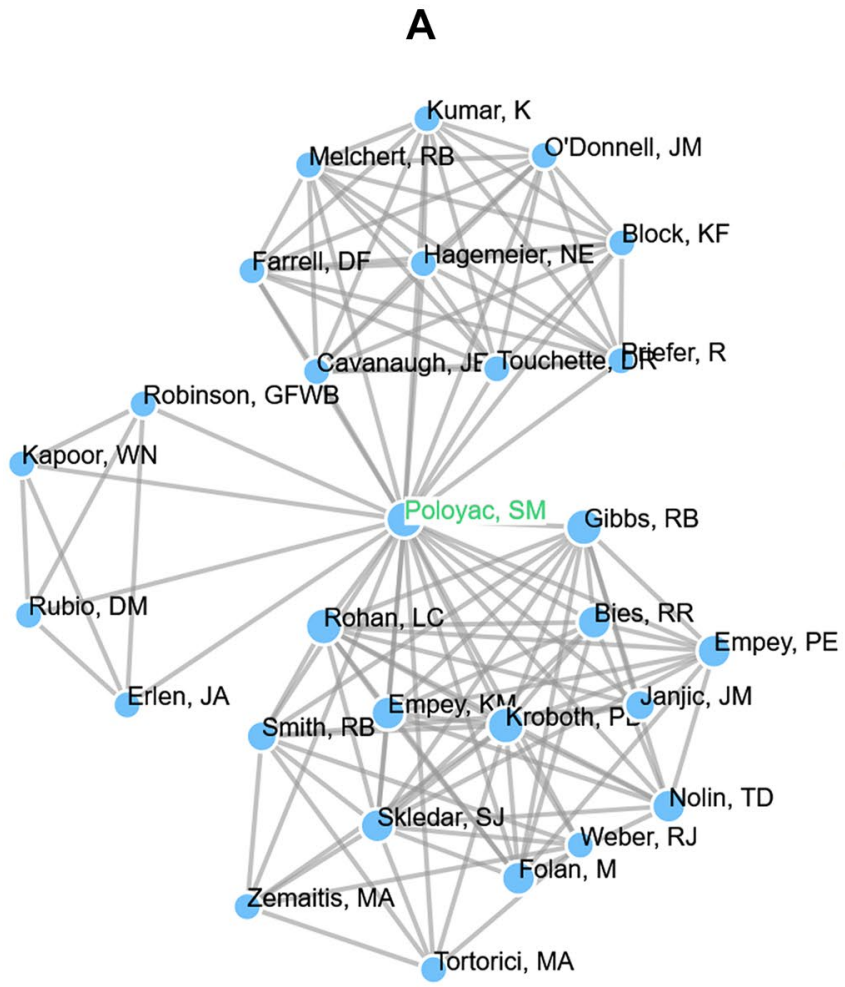

B

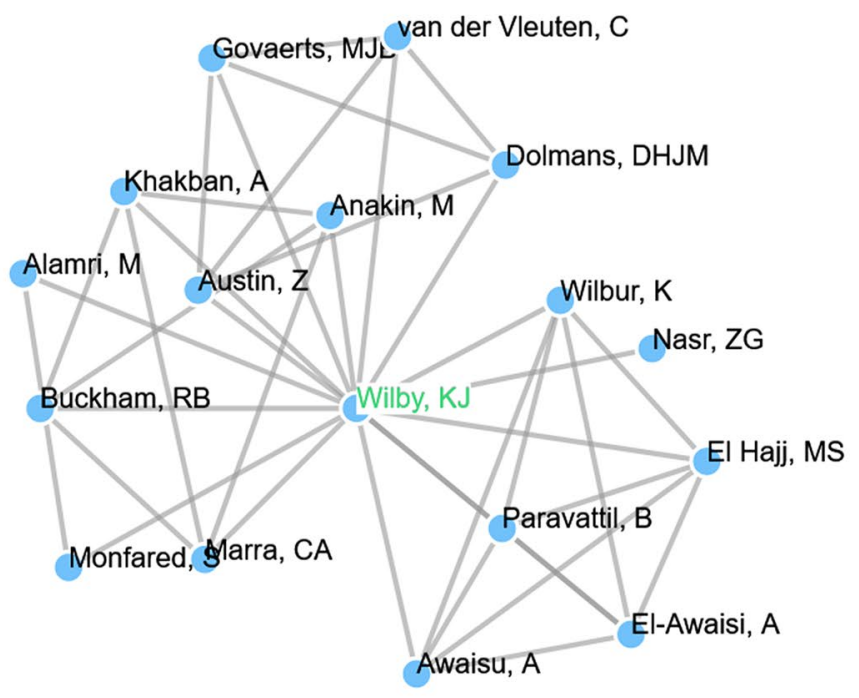

Fig. 5 Cooperation network of Poloyac S.M. (A) and Wilby K.J. (B) 


\section{Overall Profile of Citation Situations}

During the entire timespan, the analyzed publications were cited by 3264 papers (3150 without self-citations) and 3939 times cited (3774 without self-citations) of interest. The selfcitation rate was lower than $5 \%$. In average, one original paper about pharmaceutical sciences/pharmacy postgraduate education was cited by 6.73 papers ( 6.49 without selfcitations), and was cited for 8.12 times (7.78 without selfcitations). The $H$-index of this topic was 28 , which meant that there were 28 original papers having been cited for at least 28 times [19].

\section{Analysis of Cited Papers}

The 3264 papers citing the research were further analyzed, and the results were visualized. The number of cited paper and the times cited are depicted in Fig. 6A, and an evident growing trend was revealed. The times cited were slightly greater than the number of cited papers in the same year, and each paper cited less than 2 original papers. In 2000, the next year of the publication of the first 9 papers, citations started. Since 2016, the number of the citing paper and the times cited exceeded 300 per year, and both figures were over 600 in 2020. It was believed that citations in the field would continue to increase at a rapid pace in 2021 and the future.

Regarding the literature types of the citing papers, $81.07 \%$ were 'articles', $9.80 \%$ were 'reviews', and $12.22 \%$ were 'others' (Fig. 6B). Compared to the circumstances of original papers (Fig. 2A), the percentage of 'article' decreased, while that of 'review' and 'others' increased. Of note, nearly a half increment was observed in the percentage of 'review'. It indicated that the other relevant fields had been inspired by reviewing the studies of pharmaceutical sciences/pharmacy postgraduate education.

Most citing papers were contributed from the USA (1468 papers, $44.98 \%$ ), the UK (321 papers, 9.83\%), Australia (305 papers, 9.34\%), Denmark (197 papers, 6.04\%), Canada (156 papers, $4.78 \%$ ), China (117 papers, 3.58\%), Saudi Arabia (92 papers, 2.82\%), and Brazil (84 papers, 2.57\%) (Fig. 6C). Among them, the USA, the UK, Australia, Canada, China, and Saudi Arabia were also major contributing countries of the original papers. It was perceived that these countries paid relatively more attention to the field, and they were more likely to address comments on the related studies.

As for the distribution of the journals of citing papers, please refer to Fig. 4B. The periodicals American Journal of Pharmaceutical Education, Journal of Interprofessional Care, American Journal of Health System Pharmacy, BMC Medical Education, and Journal of The American Pharmacists Association that contributed a large number of original papers also published a considerable number of citing papers (714 in total, 21.88\%). Currents in Pharmacy Teaching and Learning, Pharmacy, Research in Social Administrative Pharmacy and International Journal of Clinical Pharmacy published 394 citing papers, consisting of a percentage of $12.07 \%$ of all. To sum up, the top-9 periodicals only accounted for $33.95 \%$ of citing papers, whereas the proportion of original papers published in top-9 periodicals was $60.82 \%$. It could be inferred that the distribution of journals of citing paper was more widely spreading, rather than being dominated by the top-9 journals. Additionally, it was possible that many journals which were specified in the areas other than pharmaceutical sciences/pharmacy education published the papers citing those original studies.

\section{Research Hotspot Mining}

Mining the research hotspot was a vital approach for the description of progress on pharmaceutical sciences/pharmacy postgraduate education, for it could provide valuable information about the evolving tendency of the field, and therefore guide the future investigations [20]. To this end,
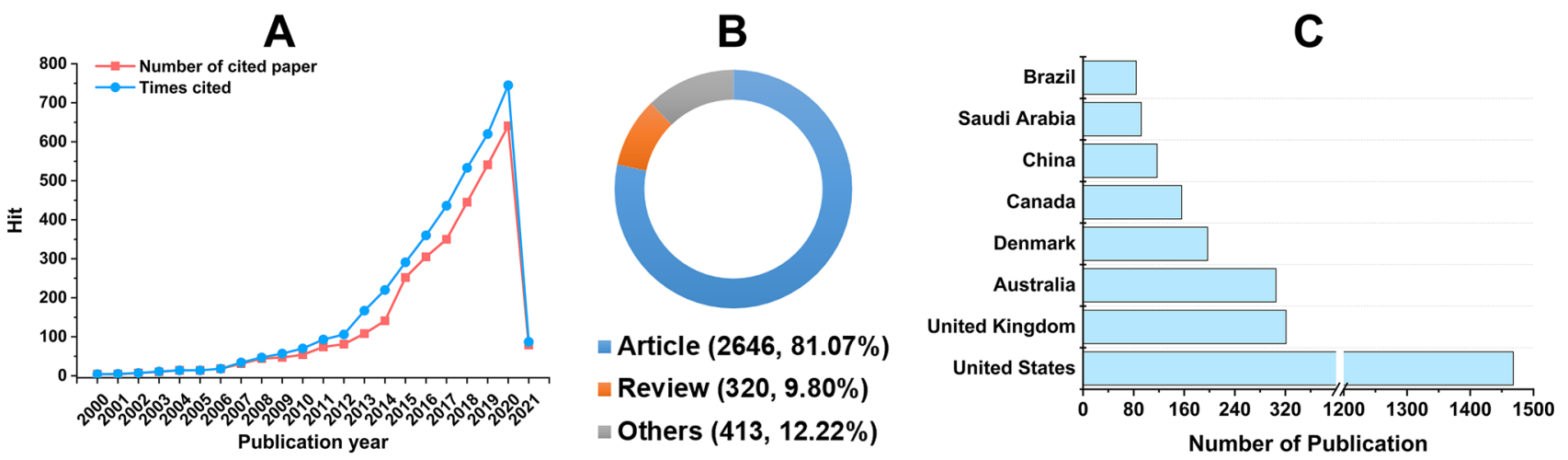

Fig. 6 Citation analysis: number of citing paper and times cited (A); distribution of literature type (B), and country/region (C) of citing papers $(n=3264)$ 
keywords, and high-frequency terms of the included papers were scrutinized.

\section{Keywords}

The keywords in 485 papers were analyzed, and the highfrequency keywords are shown in Fig. 7. The frequency of 'education', 'pharmacy', 'pharmacy education', and 'pharmaceutical education' was the highest. However, these high-frequency keywords were too general, therefore, did not provide more meaningful information. The keywords 'curriculum', 'assessment', and 'residency' also exhibited a relatively high frequency. It could be inferred that the development and revolution of curriculum, skill and capacity assessment, and the residency period management were important topics in the field. In addition, only a small proportion of papers were related with the keyword 'pharmacist'. This indicated that most papers were possibly focused on the other topics, such as postgraduate trainings.

It seemed that keywords analysis did not reflect the mastering of research hotspot as expected. Mining of highfrequency terms would help to better understand the hotspot of pharmaceutical science/pharmacy education research, and was therefore performed [21]. Of note, the minimum number of occurrences of a term was set as 20, and 120 terms (out of 11,695) met this threshold. Network visualization, time overlay visualization, and density visualization of the screened terms were conducted [22].

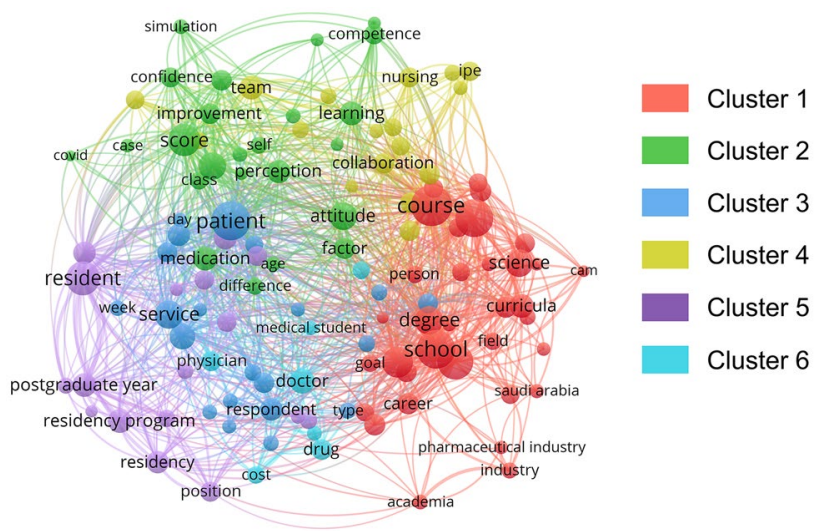

Fig. 8 Cluster network visualization of high-frequency terms $(n=120)$

\section{Cluster Network Visualization of High-Frequency Terms}

Firstly, the weights of terms were normalized by the method of association strength. The size of circle and label of a certain term were positively proportional to the weight [23]. For example, the terms 'course', 'school', 'degree', 'patient', and 'resident' are of highest weight (Fig. 8), as the in-school and in-residency cultivation of postgraduates were critical topics.

Via hierarchical cluster analysis [24], 120 terms were classified into 6 clusters (colored as red, green, blue,

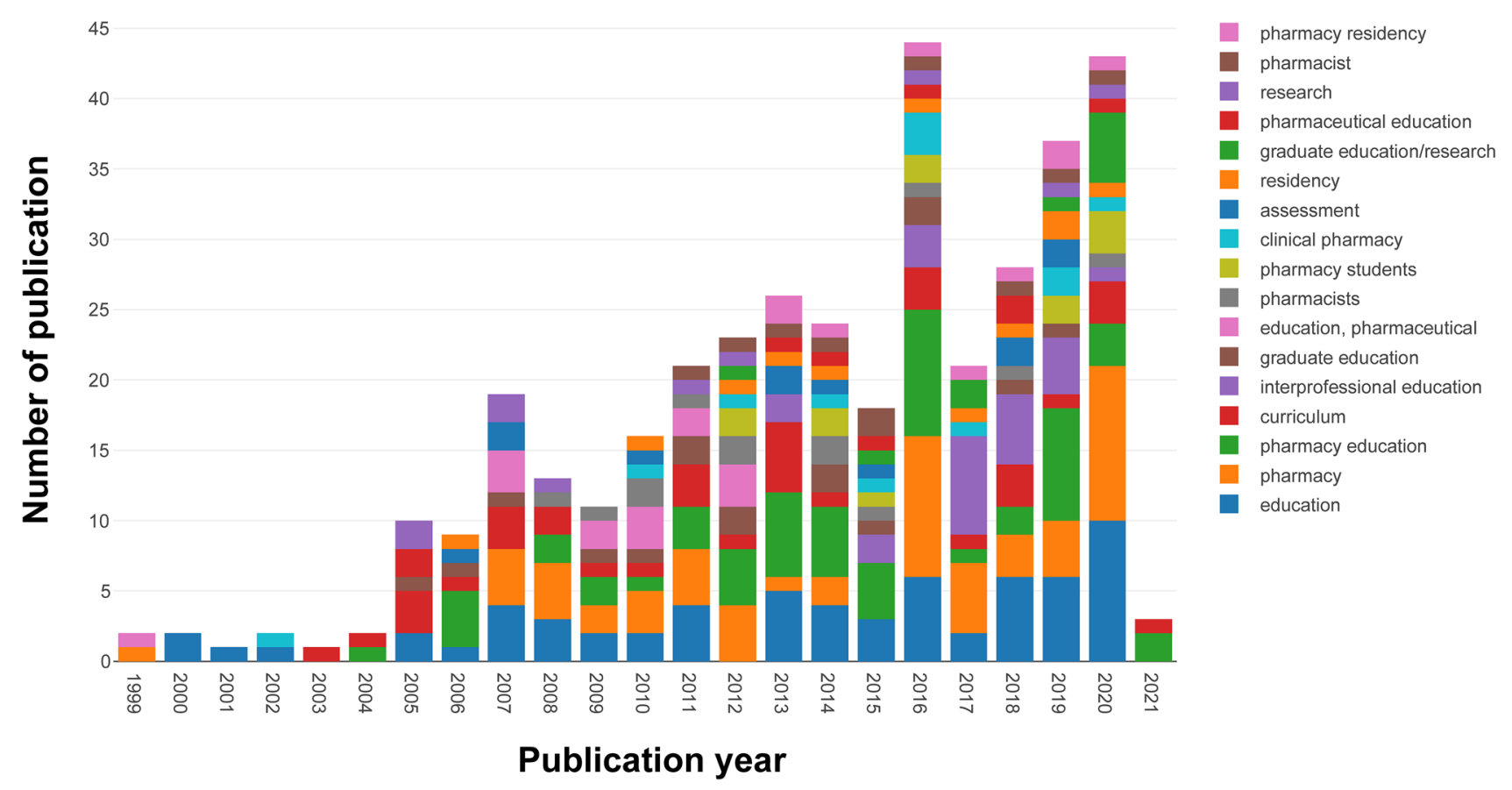

Fig. 7 Keywords frequency versus publication year $(n=485)$ 
yellow, purple, and cyan), as illustrated in Fig. 8. The significance of different clusters could be interpreted as follows.

Cluster 1: In-school education systems, representative terms are 'course', 'school', 'curricula', 'degree', 'science', 'CAM (complementary and alternative medicine)', 'person', 'goal', 'career', 'field', 'Saudi Arabia', 'industry' and 'academia'. The co-occurrence of these terms suggested that the reformation of curricula, degree awarding systems, and introduction of career-oriented goals were hot topics. Filling the gap between the demand of academia and industry was an unneglectable commission for the postgraduate education systems. Besides, the postgraduates from Saudi Arabia were important subjects for investigations.

Cluster 2: Teaching paradigm, representative terms are 'score', 'learning', 'confidence', 'attitude', 'factor', 'perception', 'competence', 'simulation', 'self', 'improvement', 'class', 'case', 'age', 'difference', 'medication' and 'COVID'. The co-occurrence of these terms implied that helping postgraduates to establish appropriate attitude the learning processes were important issues. Notably, discussions of revolution of teaching paradigms in the COVID-19 scenarios have emerged and discussed.

Cluster 3: Pharmacy services, representative terms 'patient', 'service', 'respondent', 'type', 'day' and 'week'. The co-occurrence of these terms indicated that the period of pharmacy services was a hot topic and should be included during the training of postgraduates in pharmacy.
Cluster 4: Interprofessional education (IPE), representative terms 'IPE', 'team', 'collaboration' and 'nursing'. The co-occurrence of these terms showed that interprofessional cultivation for pharmacy postgraduates was a major trend, in which a collaborative team consisting of pharmacists, clinicians, and nurses was required. This cluster also explained the reason why the Journal of Interprofessional Care published many papers of interest.

Cluster 5: Residency, representative terms 'resident', 'postgraduate year', 'residency' and 'position'. The cooccurrence of these terms reflexed that residency program was indispensable for pharmacy postgraduates.

Cluster 6: Medical systems, representative terms 'doctor', 'physician', 'medical student', 'drug' and 'cost'. The cooccurrence of these terms might be associated with the research on the education of clinical pharmacy.

In summary, the training process of pharmacy postgraduates was a research focus in the field. In contrast, that of pharmaceutical sciences postgraduates was less discussed, possibly due to the nature of the professional education for pharmacy degrees, which attracted more attention for investigators than the degrees in natural sciences.

\section{Item Density Visualization of High-Frequency Terms}

The method of Lin/log modularity was utilized for the normalization of the weights of terms [25]. The item density is visualized in Fig. 9. The density of a term was represented by color, ranging from cyan to green and red. Red color and cyan color represented that the number of associated terms
Fig. 9 Item density visualization of high-frequency terms $(n=120)$

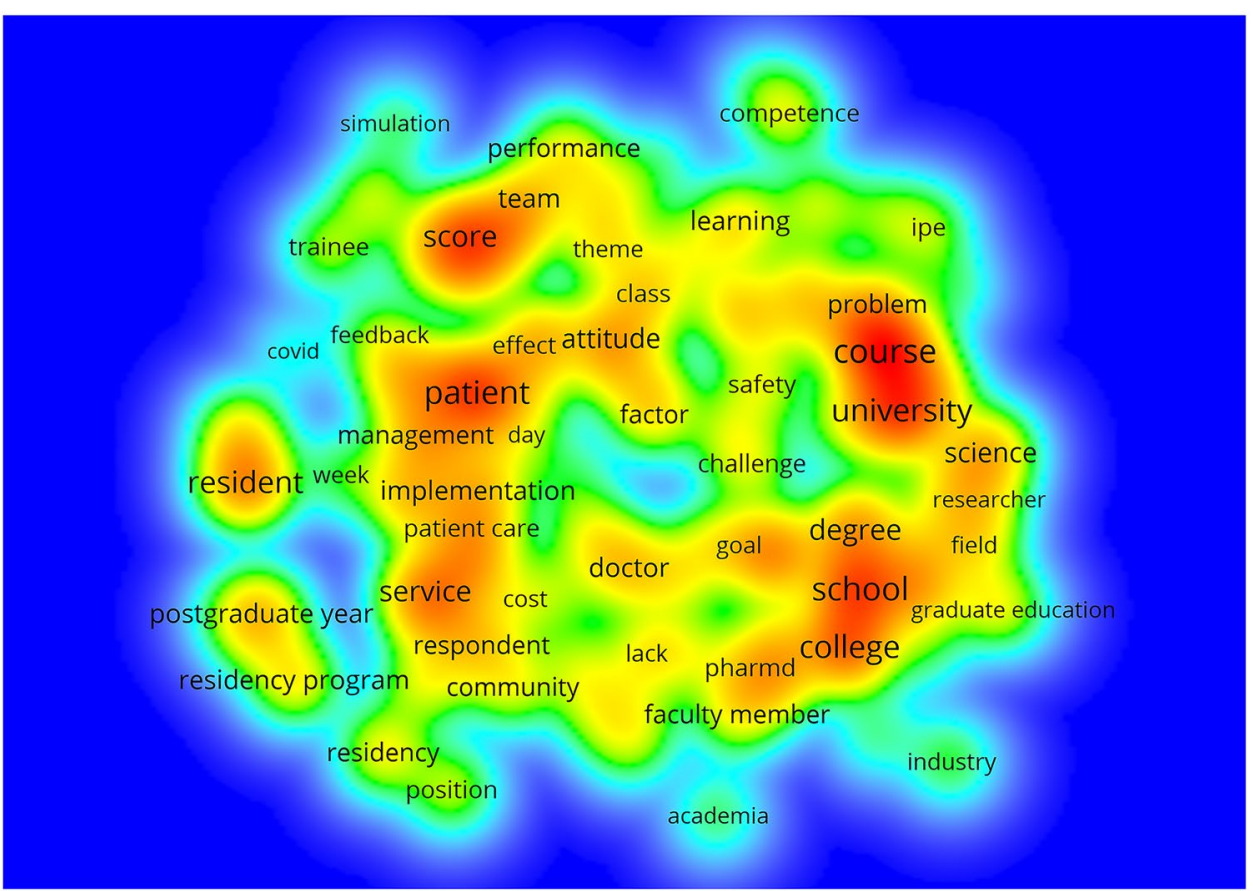


was high and low, respectively. The density could be further perceived as the degree of saturation [26].

The terms 'course', 'university', 'school', 'college', 'patient' and 'score' in red zones meant that the degree of research saturation was relatively high, while the terms like 'IPE' in the green zones were at a relatively low degree of research saturation. The zone color of terms 'academia' and 'industry' was a mix of green and cyan, and the term 'COVID' was in cyan zones, suggesting that such topics were not yet well explored.

Based on the degree of research saturation, building connections between academia and industry for pharmaceutical sciences postgraduates and constructing new modes of education under the COVID condition could be considered for future studies, in that such discussions are still insufficient currently.

\section{Time Overlay Visualization of High-Frequency Terms}

Prior to the layout, the weights of terms were normalized by the method of fractionalization. The time overlay visualization described the time evolution of high-frequency terms. The cool-warm diagram (Fig. 10) depicts the mean documented year of the terms, where warm dots and cool dots represented the recent documented years and the former years, respectively [27]. Specifically, red, gray, and blue colors represented that the average documented date of the terms was after June of 2014, between June of 2013 and June of 2014, and before June of 2013, respectively.

Typical terms for each category were shown as follows. Blue colored zones: 'course', 'school', and 'science'; Gray colored zones: 'career', 'residency', and 'PGY2 (postgraduate year two)'; Red colored zones: 'patient', 'team', and 'IPE'. It was worth mentioning that 'COVID' was located in the red colored zones as well. Moreover, 'score', a classical concept, was in the red colored zones, which indicated the indispensable role of evaluation for postgraduate education [28].

Accordingly, the time evolution of the hotspots of pharmaceutical sciences/pharmacy postgraduate education research could be divided as three stages: (1) Reformation of in-school curricula; (2) Career-oriented cultivation; (3) Interprofessional training in the new era. Seen from the last stage, the authors foresaw that in the near future,

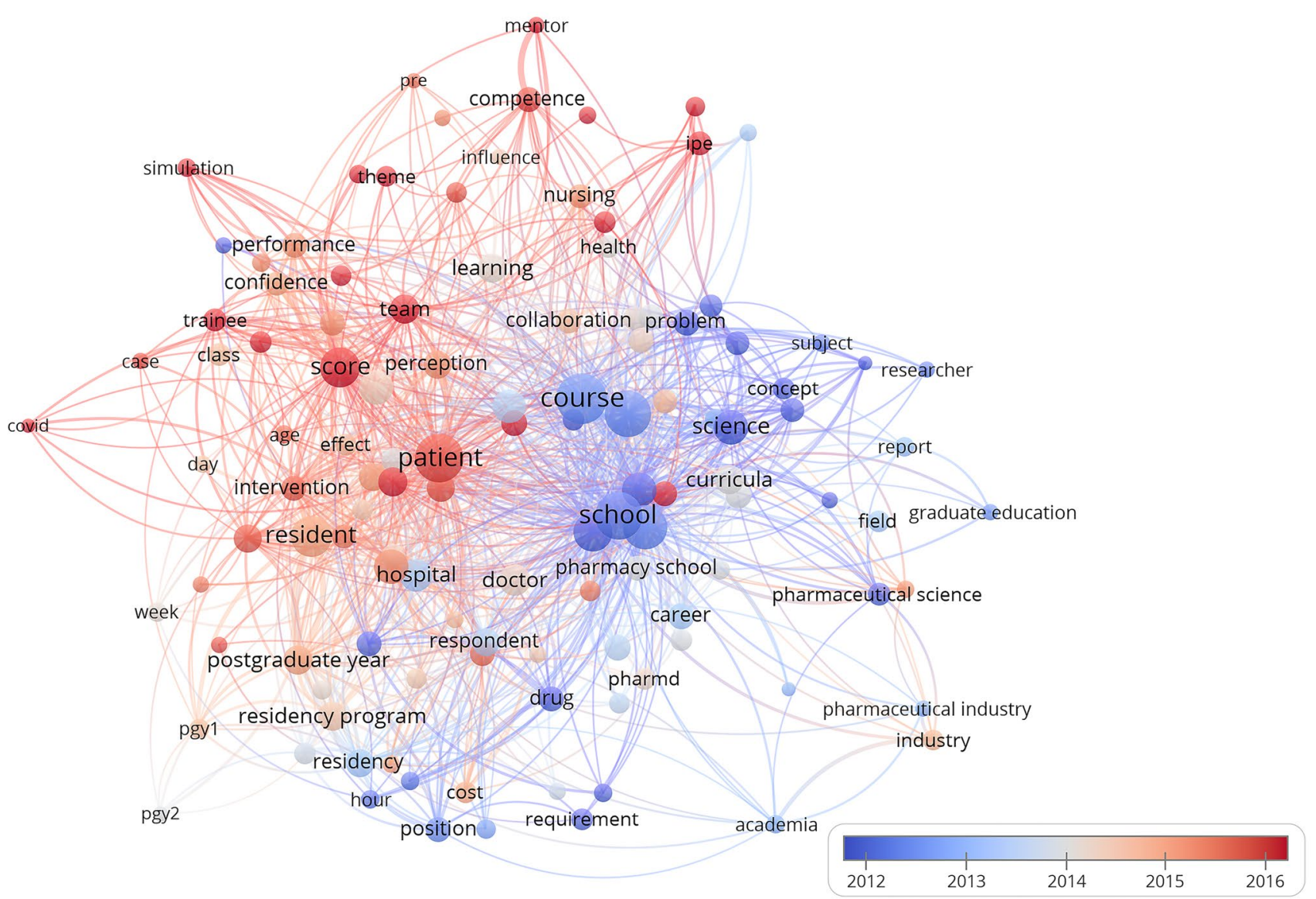

Fig. 10 Time overlay visualization of high-frequency terms $(n=120)$ 
interprofessional training of pharmacy postgraduate for the battling of COVID pandemic will become a hotspot for research.

\section{Inspiration for Pharmaceutical Sciences/Pharmacy Postgraduate Education}

Inspired by the bibliometric analysis results, some suggestions were proposed to boost the research of pharmaceutical sciences/pharmacy postgraduate education. Specifically, several possible reformed actions for governments and feasible research topics for future investigators were discussed. These suggestions were considered to be universal and might be applicable in both developed and developing countries.

\section{Promotional Actions of Government}

It is suggested that the government can conduct the following actions [29]:

1. Releasing favorable policies to encourage research programs of pharmaceutical sciences/pharmacy postgraduate education in universities and institutes. By this means, universities and institutes will be more willing to recruit investigators, and meanwhile pay more attention to the studies in this field.

2. Establishing specific funding for pharmaceutical sciences/pharmacy postgraduate education. With ample financial supports, extensive studies in-depth in the field will possibly be allowed to emerge.

3. Launching new periodicals about pharmaceutical sciences/pharmacy postgraduate education. New platforms for showcasing the research outcomes will encourage and facilitate the communications in the field.

\section{Feasible Research Topics of Investigators}

For investigators, there are several feasible research topics that might attract attentions:
1. Training of pharmaceutical sciences postgraduates. This is a relatively ignored side in the field. According to the research hotspot mining results, the current focus of the investigators is on the training of pharmacy postgraduates. Pharmaceutical sciences postgraduates are the vital labor force for academical and industrial drug discovery and development, and hence the training of them could be further emphasized.

2. Interprofessional training. For pharmacy postgraduates, interprofessional training by introducing clinical and nursing skills can help the students to understand the medical systems in the real world; for pharmaceutical sciences postgraduates, interprofessional training in chemistry, physics, engineering and artificial intelligence (AI) knowledge will help them to adapt for the modernized pharmaceutical industry.

3. Exploration of new education methods under COVID conditions. As COVID has become a long-term epidemic all across the world [30], case-based learning (CBL), problem-based learning (PBL), research-based learning (RBL), and team-based learning (TBL) regarding COVID will be helpful to reconstruct the education methods [31].

\section{Comparison with the Studies in other Fields and Previous Bibliometric Studies}

Finally, in order to dig more meanings from this study, a comparison with the studies in other fields and previous bibliometric studies on pharmaceutical sciences/pharmacy education was conducted.

\section{Comparison with the Studies in other Fields}

A quick search of the publications about postgraduate education in relatively close disciplines, viz. chemical engineering, material sciences, medicine, biology, and nanotechnology was conducted. The results are listed in Table 1. It was shown that more papers were published in the education field of medicine, materials science, and biology, which

Table 1 Basic bibliometric profiles of postgraduate education publications in pharmaceutical sciences/pharmacy and other fields

\begin{tabular}{lllllc}
\hline Education field & $\begin{array}{l}\text { Number of } \\
\text { publications }\end{array}$ & $\begin{array}{l}\text { Most fruitful } \\
\text { country/region }\end{array}$ & Journal with the most publications & Total times cited & $H$-index \\
\hline $\begin{array}{l}\text { Pharmaceutical sciences/ } \\
\text { pharmacy }\end{array}$ & 485 & USA & American Journal of Pharmaceutical Education & 3939 & 28 \\
$\begin{array}{l}\text { Chemical engineering } \\
\text { Material sciences }\end{array}$ & 223 & USA & Journal of Chemical Education & 1054 & 3790 \\
Medicine & 839 & USA & Edulearn Proceedings & 54,617 & 28 \\
Biology & 4363 & USA & Academic Medicine & 3962 & 29 \\
Nanotechnology & 629 & USA & Biochemistry and Molecular Biology Education & 373 & 11 \\
\hline
\end{tabular}


Table 2 Overview of the previous bibliometric studies on pharmaceutical sciences/pharmacy education

\begin{tabular}{|c|c|c|c|c|c|c|}
\hline Ref. no & Publication year & Survey database & Survey timespan & Research focus & $\begin{array}{l}\text { Main } \\
\text { conclusion }\end{array}$ & Reference \\
\hline 1 & 2010 & $\begin{array}{l}\text { China Biology Medicine } \\
\text { disc (CBMdisc) }\end{array}$ & 1978-2010 & $\begin{array}{l}\text { Clinical pharmacy } \\
\text { education in China }\end{array}$ & $\begin{array}{l}\text { Clinical pharmacy } \\
\text { education in China } \\
\text { undergone four } \\
\text { development stages }\end{array}$ & [32] \\
\hline 2 & 2013 & $\begin{array}{l}\text { Science Direct, Springer } \\
\text { Links, Medline, PubMed }\end{array}$ & $\sim 2011$ & $\begin{array}{l}\text { Pharmacy education in } \\
\text { low- to middle-income } \\
\text { countries }\end{array}$ & $\begin{array}{l}\text { There was a dominance } \\
\text { of commentary and } \\
\text { viewpoint papers while } \\
\text { empiric literature was } \\
\text { scarce }\end{array}$ & [33] \\
\hline 3 & 2015 & $\begin{array}{l}\text { Wanfang, CQVIP, China } \\
\text { National Knowledge } \\
\text { Infrastructure (CNKI) }\end{array}$ & $\sim 2013$ & $\begin{array}{l}\text { Discipline construction } \\
\text { of clinical pharmacy in } \\
\text { Chinese universities }\end{array}$ & $\begin{array}{l}\text { The education paradigm } \\
\text { of clinical pharmacy } \\
\text { had witnessed a great } \\
\text { transformation }\end{array}$ & [34] \\
\hline 4 & 2018 & SciVerse Scopus & 2000-2016 & $\begin{array}{l}\text { Worldwide pharmacy } \\
\text { education research }\end{array}$ & $\begin{array}{l}\text { Research interest of } \\
\text { pharmacy education was } \\
\text { growing, and multi- } \\
\text { professional learning and } \\
\text { role of social media had } \\
\text { received high number of } \\
\text { citations }\end{array}$ & [35] \\
\hline
\end{tabular}

were 9.0, 1.8, and 1.3-folds of those published in pharmaceutical sciences/pharmacy. Less publications were found in chemical engineering and nanotechnology education, equal to $46.0 \%$ or $19.6 \%$ of the numbers in pharmaceutical sciences/pharmacy. USA was the country/region that published the highest number of studies. The journals with the most publications were all educational journals. For medicine education, the numbers of total times cited and $H$-index were significantly higher than other fields. The number of total times cited and $H$-index in the fields of material sciences and biology were similar to pharmaceutical sciences/ pharmacy, while those numbers of chemical engineering and nanotechnology were remarkably lower.

According to the above results, the degree of attention paid to postgraduate education research varied with different disciplines. Based on the fruitful research outcomes in the field of medicine, the investigators in pharmaceutical sciences/ pharmacy postgraduate education were recommended to learn from the researchers in medicine postgraduate education.

\section{Comparison with Previous Bibliometric Studies}

A few (4) previously published bibliometric studies with similar aims were found. The small number indicated that there were much to be explored in this field. The main information of these studies is summarized in Table 2.

Overall, neither paper analyzed the progress in both pharmaceutical sciences and pharmacy fields, nor of those 4 papers focused on the postgraduate education. Furthermore, as shown in Table 2, reference 1 to 3 discussed the development of (clinical) pharmacy education in a small number of countries, only the reference 4 showed a global vision for the study. Additionally, those studies were conducted before 2016, which urges a more updated and comprehensive study in the field. It is noted that a variety of databases (like Medline and CNKI) were used in the previous studies, although they are less popular or in the language other than English.

\section{Conclusion}

The research progress on pharmaceutical sciences/pharmacy postgraduate education was analyzed in bibliometric perspective in this work. 485 papers were screened in the literature survey. In general, the numbers of publications were growing along with year, and the main type of literature was article. Institutions in the USA were the major contributors; however, in terms of authorship, each author contributed averagely no more than 5 papers. Most papers were composed in English, and published in education-specified, medical system-related and fundamental research-based journals. It should be noted that only a small percentage of the studies were sponsored by grants. As for the citation analysis, the numbers of the citing papers were increasing with time, and the distribution attributes of the cited papers were similar to those of original papers. The research hotspots laid in the training of pharmacy postgraduates, and the establishment of new education paradigms in the COIVD time was a recent research focus.

It was anticipated that the bibliometric analysis could help to catalyze the promotional actions of government and feasible research topics of investigators regarding the 
studies upon pharmaceutical sciences/pharmacy postgraduate education.

Supplementary Information The online version contains supplementary material available at https://doi.org/10.1007/s12247-021-09611-z.

Author Contribution Zhengwei Huang, data analysis and writing; Xuejuan Zhang, literature survey and visualization; Linjing $\mathrm{Wu}$, suggestion proposing, manuscript formatting and manuscript revision; Ping $\mathrm{Hu}$, manuscript polishing and revision; Ying Huang and Chuanbin Wu, project supervision and manuscript polishing; Xin Pan, conceptualization, fund-seeking and proof-reading.

Funding The authors greatly appreciated the funding from the National Natural Science Foundation of China, under grant nos. 82073774 and 81973263

\section{Declarations}

Ethics Approval and Consent to Participate NA.

Conflict of Interest The authors declare no competing interests.

\section{References}

1. Drennen JK. Healthcare reform: a risk to pharmaceutical innovation? J Pharm Innov. 2009;4(2):92-3.

2. Wang Y, Yu Q, Zhu K. Drugs supply and pharmaceutical care management practices at a designated hospital during the COVID19 epidemic. Res Soc Admin Pharm. 2021;17(1):1978-83.

3. Kuo SC, Ou HT, Wang CJ Managing medication supply chains: lessons learned from Taiwan during the COVID-19 pandemic and preparedness planning for the future. J Am Pharm Assoc. 2021;61(1):E12-E5.

4. Chang S. Supporting expansive learning in preservice bilingual teachers' zone of proximal development of the activity system: an analysis of a four-field model trajectory. Prof Dev Educ. 2021;6.

5. Wang J, Hu XM, Liu J, et al. Pharmacy students' attitudes towards physician-pharmacist collaboration: intervention effect of integrating cooperative learning into an interprofessional team-based community service. J Interprof Care. 2016;30(5):591-8.

6. Tawfiq AM, Alomar MJ, Hassan N, et al. Nationwide survey on attitudes and perceived barriers toward provision of pharmaceutical care among final year undergraduate pharmacy students in the United Arab Emirates. PLoS One. 2021;16(2):e0246934.

7. Liu WS. Caveats for the use of Web of Science Core Collection in old literature retrieval and historical bibliometric analysis. Technol Forecast Soc Change. 2021;172.

8. Hafeez DM, Jalal S, Khosa F. Bibliometric analysis of manuscript characteristics that influence citations: a comparison of six major psychiatry journals. J Psychiatr Res. 2019;108:90-4.

9. Zhai K, Ma W, Huang T. Hot spots and trends in knee revision research since the 21 st century: a bibliometric analysis. Ann Transl Med. 2021;9(5):388.

10. van Eck NJ, Waltman L. Software survey: VOSviewer, a computer program for bibliometric mapping. Scientometrics. 2010;84(2):523-38.

11. Gao Y, Qu B, Shen Y, et al. Bibliometric profile of neurogenic bladder in the literature: a 20-year bibliometric analysis. Neural Regen Res. 2015;10(5):797-803.
12. Dehghanbanadaki H, Seif F, Vahidi Y, et al. Bibliometric analysis of global scientific research on coronavirus (COVID-19). Med J Islam Repub Iran. 2020;34-51.

13. Jiang JH, Liu Y, Wang YJ, et al. Clinical pharmacy education in China. Am J Pharm Educ. 2011;75(3):57c.

14. Alzahrani R, Harris E. Biopharmaceutical revolution in Saudi Arabia: progress and development. J Pharm Innov. 2020;16(1):110-23.

15. Cao S, Huang H, Xiao M, et al. Research on safety in home care for older adults: a bibliometric analysis. Nurs Open. 2021;8(4):1720-30.

16. Weggemans MM, van Dijk B, van Dooijeweert B, et al. The postgraduate medical education pathway: an international comparison. GMS J Med Educ. 2017;34(5):Doc63.

17. Williams JP. What educational psychology means to me: the journey of a reading researcher. Educ Psychol-Us. 2021;56(1):18-28.

18. Liu XZ, Zhang JS, Guo C. Full-text citation analysis: a new method to enhance scholarly networks. J Am Soc Inf Sci Technol. 2013;64(9):1852-63.

19. Brito R, Navarro AR. The inconsistency of h-index: a mathematical analysis. J Informetr. 2021;15(1).

20. Ho L, Goethals P. Research hotspots and current challenges of lakes and reservoirs: a bibliometric analysis. Scientometrics. 2020;124(1):603-31.

21. dos Santos BS, Steiner MT, Fenerich AT, Lima RH. Data mining and machine learning techniques applied to public health problems: a bibliometric analysis from, et al 2009 to 2018. Comput Ind Eng. 2019;138.

22. Sood SK, Kumar N, Saini M. Scientometric analysis of literature on distributed vehicular networks: VOSViewer visualization techniques. Artif Intell Rev. 2021;8.

23. Zheng JT, Liu NC. Mapping of important international academic awards. Scientometrics. 2015;104(3):763-91.

24. van Eck NJ, Waltman L. Citation-based clustering of publications using CitNetExplorer and VOSviewer. Scientometrics. 2017;111(2):1053-70.

25. Dinić BM, Jevremov T. Trends in research related to the Dark Triad: a bibliometric analysis. Curr Psychol. 2019.

26. Guleria D, Kaur G. Bibliometric analysis of ecopreneurship using VOSviewer and RStudio Bibliometrix, 1989-2019. Library Hi Tech. 2021.

27. Xing D, Zhao Y, Dong S, Lin J. Global research trends in stem cells for osteoarthritis: a bibliometric and visualized study. Int J Rheum Dis. 2018;21(7):1372-84.

28. Wesling M, Schumacher C, Liu JJ, et al. Postgraduate year two ambulatory care residency program evaluation with a tool for assessing ambulatory care pharmacist practice. J Am Coll Clin Pharm. 2021.

29. Mao DL, Zheng Q. Encouraging an environment for pharmaceutical R\&D in China. J Pharm Innov. 2009;4(3):152-4.

30. Yang Q, Yi C, Vajdi A, et al. Short-term forecasts and long-term mitigation evaluations for the COVID-19 epidemic in Hubei Province China. Infect Dis Model. 2020;5:563-74.

31. Giuliano C, Martirosov AL, Lipari M, et al. Incorporating verbal defense into problem-based learning. Curr Pharm Teach Lea. 2021;13(2):109-15.

32. Liu XF, Yang MS. Bibiometric analysis of the clinical pharmacy education. China Med Heral. 2010;7(27):98-100.

33. Babar ZUD, Scahill SL, Akhlaq M, et al. A bibliometric review of pharmacy education literature in the context of low- to middleincome countries. Curr Pharm Teach Learn. 2013;5(3).

34. Hao GX. Bibliometric analysis on the clinical pharmacy education. Pharm Educ. 2015;31(01):27-33.

35. Sweileh WM, Al-Jabi SW, Zyoud SH, et al. Bibliometric analysis of literature in pharmacy education: 2000-2016. Int J Pharm Pract. 2018;26(6):541-9.

Publisher's Note Springer Nature remains neutral with regard to jurisdictional claims in published maps and institutional affiliations. 